\title{
SISTEMATIZACION DE LA EXPERIENCIA EN LA FORMACIÓN DE ASISTENTE TÉCNICO EN ATENCIÓN PRIMARIA EN SALUD ${ }^{1}$
}

Olga Sánchez-Charpentier ${ }^{2}$

Institución: Escuela de Enfermería de la Universidad de Costa Rica

\section{COMO CITAR}

\section{RESUMEN}

Este artículo presenta la sistematización de la experiencia vivida por la Escuela de Enfermería respecto de la formación de Asistentes Técnicos de Atención Primaria de Salud (ATAPS) durante el periodo 2003 - 2011. Inicialmente, en Costa Rica y en los años 60, surgen los primeros grupos de asistentes en atención primaria; de su capacitación se encargaban los y las enfermeras del Programa de Atención Primaria del Ministerio de Salud y esta tomaba entre tres y cinco meses. A partir de los años 90 con la Reforma del Sector Salud, se forman los Equipos Básicos de Atención Integral en Salud (EBAIS), por lo que se crea la necesidad de capacitar integralmente este recurso humano. Se recopiló y analizó la información obtenida a través de entrevistas y cuestionarios y se elaboró una revisión documental acerca de la atención primaria en Costa Rica y la formación de Asistentes Técnicos de Atención Primaria de Salud en el periodo del 2003 al 2011 con el fin de sistematizar los datos. Durante el periodo 2003-2011, la población formada como Asistente Técnico en Atención Primaria en Salud fue de 420 personas y estuvo compuesta principalmente por mujeres solteras de 18 a 30 años, procedentes de San José, con un buen rendimiento académico y que se costearon sus estudios con la Cooperativa de Ahorro y Préstamo (COONAPE) Se concluye que la Escuela de Enfermería se proyecta a la población nacional por medio de la acción social formando recurso humano de calidad en Atención Primaria en Salud tanto para el sector público como privado.

Palabras clave: atención-primaria, asistentes-técnicos, Enfermería, EBAIS

\footnotetext{
${ }^{1}$ Fecha de recepción: 20 de noviembre 2011

Fecha aceptación: 15 de enero 2012

${ }^{2}$ Enfermera. Profesora de la Universidad de Costa Rica. Magister en Enfermería en Salud Laboral. Correo electrónico: marysacha@gmail.com
} 


\title{
SYSTEMATIZATION EXPERIENCE IN THE TRAINING TECHNICAL ASSISTANT IN PRIMARY HEALTH CARE ${ }^{1}$
}

Olga Sánchez-Charpantier ${ }^{2}$

Institution: School of Nursing, University of Costa Rica

\section{CITED}

\begin{abstract}
This article presents the systematization of the experience of the School of Nursing on the formation of Technical Assistants in Primary Health Care (ATAPS) during the period 2003 to 2011. Initially, in Costa Rica and in the 60 arise the first groups of workers in primary care were in charge of training the nurses and Primary Care Program of the Ministry of Health and this would take three to five months. From the 90's with the Health Sector Reform, they form the Basic Teams for Integral Health (EBAIS), thus establishing the need to train fully this human resource. Collected and analyzed information obtained through interviews and questionnaires and produced a documentary review about primary care in Costa Rica and the formation of Technical Assistants in Primary Health Care in the period from 2003 to 2011 in order to systematize the data. During the period 2003-2011, the population formed as Technical Assistant in Primary Health Care was 420 people and consisted mainly of single women ages 18 to 30, from San Jose, with a good academic standing and were paid for their cooperative studies in the institution for Savings and Loan (COONAPE). It is concluded that the School of Nursing is projected to the national population through social action forming human resources quality in primary health care for both public and private sectors.
\end{abstract}

Keywords: primary-care, technical-assistants, nurses, EBAIS

\footnotetext{
${ }^{1}$ Date of reception: November 20, 2011

Date of acceptance: January 15,2012

${ }^{2}$ Enfermera. Profesora de la Universidad de Costa Rica. Magister en Enfermería en Salud Laboral. E mail: marysacha@gmail.com
} 


\section{INTRODUCCIÓN}

Ante la gran cantidad de vivencias prácticas derivadas del trabajo realizado por la Escuela de Enfermería de la Universidad de Costa Rica respecto de la formación de Asistentes Técnicos en Atención Primaria durante el periodo 2003 - 2011, fue necesario sistematizarla, es decir, interpretar la experiencia vivida de una manera crítica, ya que a partir de su ordenamiento y reconstrucción se descubre y explicita la lógica del proceso vivido, los factores que han intervenido en dicho proceso, la relación entre estos y el porqué lo han hecho de ese modo. (Jara, 1998)

En 1978 nace el concepto y la definición de la Atención Primaria en Salud (APS), en una reunión convocada por la Organización Mundial de la Salud (OMS) y el Fondo de las Naciones Unidas para la Infancia (UNICEF) en Alma Ata, antigua URSS. (Vargas, 2006); para finales del siglo XX, la Organización Mundial de la Salud (OMS) y la Oficina Panamericana de la Salud (OPS) hacen un relanzamiento de la Atención Primaria e instan a todos los países a que retomen con mayor fuerza este tema, ya que la meta establecida de Salud para Todos en el Año 2000 estuvo muy alejada de sus objetivos en numerosos países del orbe. (OPS, 2004)

En Costa Rica, a finales de los años ochenta había una sensación en los dirigentes políticos y en los técnicos del sistema de salud de que el modelo de atención primaria ya estaba agotado, por lo que se realizaron varios estudios y evaluaciones del sistema, cuyas conclusiones demostraron graves deficiencias. Debido a esto, en 1989 se organizó el Primer Foro Nacional de Salud con la participación de funcionarios del Ministerio de Salud, de la Caja Costarricense del Seguro Social (CCSS) y representantes gremiales; en este foro se concluye que existe la necesidad de iniciar una profunda reforma del modelo de atención de salud, dada la ineficiencia del sistema y la insatisfacción de los usuarios y de los prestatarios de los servicios. (Vargas, 2006)

Debido a lo anterior, alrededor de los años noventa, Costa Rica asumió el Modelo Readecuado de Salud, con el principal propósito de brindar estrategias de salud integral a las regiones que la constituyen, de allí que a mediados de la década se estaban atendiendo alrededor de 1.407 .621 personas y para finales de la década se incrementó la cobertura a la mayor cantidad de la población. (Proyecto Estado de la Nación, 2005)

A partir de la Reforma del Sector Salud se crean los Equipos Básicos de Atención Integral en Salud (EBAIS), los cuales están constituidos por un médico, un auxiliar de enfermería y un Asistente Técnico de Atención Primaria en Salud (ATAPS), quien funciona bajo el principio de trabajo en equipo. El ATAPS ejecuta labores de asistencia técnica a nivel domiciliar, en el área de salud rural, periurbana y urbanas marginales. (Ayala, Carvajal, Cercome y Arce, 1999). Además, los ATAPS establecen contacto directo con los individuos, las familias y la comunidad para satisfacer las necesidades básicas que contribuyen a su bienestar tales como programas de vacunación, atención al niño y la niña menores de un año, trabajo escolar en la población infantil mayor de 6 años, atención materno infantil, entre otros . (Garro, 1997 y González, 1992). Estas prácticas sustentan la estrategia de atención primaria y junto con el equipo de salud (EBAIS) y el equipo de apoyo, son los responsables de la atención integral a la salud de las personas en un espacio poblacional. (Méndez y Parada, 2000) 
Alrededor de los años setenta, en Costa Rica se inició la conformación de los primeros grupos de asistentes en atención primaria en las zonas rurales. A las personas interesadas en desempeñar esta labor no se les solicitó ningún requisito para ingresar y su capacitación se extendía por tres meses. En 1974, se amplía el periodo de formación a cinco meses y el proceso estaba a cargo de los profesionales de enfermería del Programa de Atención Primaria del Ministerio de Salud, quienes también se encargaban de supervisarlos. (Barrantes, 2000)

Con la creación de los EBAIS, surge la necesidad de capacitar de forma integral al Asistente Técnico de Atención Primaria en Salud (ATAPS), por lo que en 1984 el Ministerio de Salud inicia las gestiones ante la Universidad de Costa Rica para obtener el aval y acreditación para los cursos de capacitación (Montero y Quirós, 2003). A partir del año 1999, y por medio del Programa de Extensión Docente de la Escuela de Enfermería y la Vicerrectoría de Acción Social, se inicia el curso de formación de este recurso humano con el personal de las Áreas de Salud de las comunidades de Tirrases y Curridabat. (Meza citado por Montero y Quirós, 2003). Para la capacitación, la Escuela de Enfermería cuenta con la participación del personal docente, así como de otras unidades académicas de la universidad e instituciones públicas que aportan conocimientos en las diferentes temáticas durante la formación de este recurso humano en salud.

El objetivo de esta sistematización fue recuperar la experiencia de la formación de los ATAPS y hacer un análisis crítico del proceso para obtener nuevos conocimientos en el campo de la Atención Primaria en Salud.

\section{MATERIALES Y MÉTODO}

Como parte de la sistematización, se revisaron documentos que abordan la temática de la atención primaria en el país y la formación de ATAPS.

Para conocer y comprender la experiencia de la Escuela de Enfermería de la Universidad de Costa Rica en la formación de los ATAPS, se utilizó la sistematización del proceso vivido, ya que es un proceso reflexivo sobre la experiencia que se realiza mediante aproximaciones sucesivas. Su utilidad radica en ser insumo para la siguiente experiencia profesional, al comunicar logros, obstáculos y lecciones aprendidas, por lo que enriquece la práctica en sí y, por ende, la respuesta institucional ante las demandas de la sociedad. (Arce, 1998)

Los instrumentos utilizados que facilitaron la generación y recuperación de la información desarrollada en la experiencia fueron: a) Una entrevista semiestructurada donde se describen los aspectos sociodemográficos, como son la edad, sexo, lugar de procedencia, las expectativas que poseen, las experiencias previas en el área de salud, estudios realizados, la disponibilidad de tiempo, las estrategias económicas y familiares para realizar el curso; b)Entrevistas abiertas para conocer la experiencia de los y las estudiantes durante la formación; c) El análisis de los informes que realizaron los docentes responsables del curso en relación con el rendimiento académico de los participantes del año 2003 hasta el año 2011. 
Los estudiantes realizaron el curso de ATAPS en el periodo 2003 - 2011 y el grupo estuvo integrado por 420 personas. En cada semestre, los grupos tuvieron de 12 a 33 personas. A la fecha se han graduado 18 generaciones de estudiantes.

\section{Consideraciones éticas}

Respecto de la confidencialidad del estudiantado participante en los cursos, se mantuvo el anonimato de las instituciones involucradas y la retroalimentación del proceso en general.

\section{RESULTADOS}

A partir de la entrevista semiestructurada se determinaron las características y aspectos sociodemográficos del total de estudiantes, a continuación se presentan los datos.

Del total de 420 personas graduadas, 70\% (294) son mujeres y 30\% (126) son hombres. Respecto del estado civil, el $60 \%$ (252) no se ha casado, mientras que un 25\% (105) sí lo está; un 7.1\% (30) se ha divorciado; un $1.9 \%$ (8) se encuentra separado; $1 \%$ (4) viudo y un 5\% (21) vive en unión libre.

En cuanto a las edades, un 36.9\% (155) se encuentra entre los 18 y 20 años, hecho que coincide con que dicho grupo recién se ha graduado de secundaria; de 21 a 30 años un 40.7\% (171); de 31 a 40 años, 16.7\% (70)

En relación con la forma de sufragar el costo del curso, un 23.1\% (97) lo hizo por medio de préstamo con CONAPE exclusivamente; un 10\% (42) recibió ayuda de CONAPE, pero también utilizó recursos propios, un $36.2 \%$ (152) lo hizo solo con recursos propios o brindados por familiares y un 30.7\% (129) fue becado por distintas Áreas de Salud.

Acerca del lugar de procedencia o residencia por provincia, un 59 \% (248) provienen de San José; un 15.9\% (67), de Alajuela; un 10\% (42), de Heredia; 12\% (50), de Cartago; 2.4\% (10), de Guanacaste; 0.2\% (1), de Puntarenas y $0.5 \%$ (2), de Limón.

Al finalizar el curso el rendimiento académico fue el siguiente: $0.5 \%$ (2) obtuvo notas menores a 7.0 (quienes perdieron el curso); la calificación de un 44\% (185) de los estudiantes, estuvo entre 7.0 y 8.0 ; un 40\% (168), entre 8.1 y 9.0; y un 15.5\% (65) de los estudiantes, obtuvo entre 9.1 y 10.

Por otra parte, lo que los motiva a llevar el curso de ATAPS es principalmente la superación personal y profesional, además de que puede ser un escalón para estudios superiores.

En relación con la información acerca del grado de satisfacción con el curso, los estudiantes afirmaron sentirse satisfechos (as) de las temáticas que abarca el curso, además de la calidad de los docentes que los imparten, aunque en algunos aspectos quisieran mayor profundidad en los contenidos, por ejemplo en aspectos referentes a las técnicas de enseñanza, a la drogodependencia, sobre salud sexual y salud reproductiva 
enfermedades crónicas, la salud bucodental, sobre los adultos mayores y la práctica de vacunación; las recomendaciones fueron realizadas a pesar de que están conscientes de que es un curso técnico, ya que consideran que dichos conocimientos son necesarios en su futuro ejercicio, una vez finalizado su campo clínico estudiantil.(Información obtenida a partir del instrumento de evaluación de los cursos del Programa de Extensión Docente, UCR)

Durante el periodo 2003 - 2011, el personal docente a cargo informó que el rendimiento de los estudiantes era suficiente, bueno y muy bueno, además que alcanzaron las destrezas necesarias para el desarrollo técnico en su desempeño en el primer nivel de atención.

\section{DISCUSIÓN}

Desde 1999, con la formación de ATAPS la Escuela de Enfermería ha dado la oportunidad a aproximadamente 1000 personas, ya que cada año se acercan entre 50 y 100 interesadas (os) en iniciar estudios en atención primaria. Sin embargo, de las y los participantes, solo entre 35 y 65 aspirantes concluyen el curso, debido a que tienen dificultades financieras, familiares o de reubicación geográfica. Además, muchos no conocen bien el perfil del ATAPS y, por ello cuando se describe cómo será el trabajo de campo y lo que este conlleva, dígase cambios climáticos, amenazas físicas y sociales, problemas ergonómicos al cargar sus equipos y materias indispensables para la visita domiciliaria, obliga a los oferentes a hacer un análisis concienzudo de sus capacidades para formarse como ATAPS.

Los resultados demostraron que la mayoría de personas que realizan el curso está representada por mujeres, máxime si se tiene en cuenta que la situación económica y los derechos de las mujeres las han llevado al ámbito laboral, cambio que les permite aportar dinero al grupo familiar para así propiciar mejores oportunidades de vida para su familia (Lagarde, 1992)

Las edades de las y los participantes son variables; se destaca las y los jóvenes recién graduados de secundaria que desean realizar el curso para posteriormente poder costear una carrera profesional que de otro modo no podrían hacerlo, según refieren en las entrevistas. Los de mayor edad señalan otros factores que los han motivado a realizar el curso, entre estos están el establecer una familia, falta de estudios que les dificulta obtener un trabajo, mejorar su situación económica, entre otros buscan realizar un curso en poco tiempo con el que puedan solventar las necesidades de sus hogares y les permita acceder a las garantías sociales. (Guillén, 1997).

Según refieren las personas mayores de 40 años han trabajado en varios lugares y en distintas actividades, a pesar de ello no han logrado una estabilidad laboral, mas ahora consideran que podrían alcanzarla, dado que al formarse como ATAPS ven la posibilidad de establecerse y asegurarse un futuro para sí y para sus familias. Vemos que tienen las mismas expectativas que los más jóvenes, aunque el incorporarse al ámbito laboral se les dificulta un poco más por su edad, (Jiménez, Montero, Sánchez, y Tenorio, 2005). A pesar de esto, la mayoría logra incorporarse en alguna Área de Salud. 
En lo que respecta al estado civil, son más los que se encuentran solteros y solteras (relacionado con la edad); quienes se encuentran casadas o en unión libre ven la posibilidad de dar apoyo económico al núcleo familiar y realizarse personalmente; en cuanto a las y los que se encuentran divorciados(as) o separadas, las mujeres refirieron sentirse sin el apoyo económico de su pareja por diversas situaciones, por lo que ven la necesidad de buscar una oportunidad de sustento para su familia y una realización personal que fue truncada al casarse o al tener hijos.

En cuanto a la forma de pago, la mayor cantidad de participantes costean el curso por su propia cuenta, ya sea mediante la ayuda de sus padres, esposos o porque poseen algún ahorro, mientras que otros lo combinan con un préstamo en Consejo Nacional de Préstamos para la Educación, CONAPE, único medio a través del cual algunas pueden sufragar los gastos que implica la formación como ATAPS. Cabe mencionar que el autofinanciamiento representa una ventaja, ya que permite mayor libertad de movimiento en el ámbito laboral, al elegir el lugar que le sea más accesible, rentable y favorable para sus intereses y sin la presión de una beca.

En relación con el financiamiento, también están quienes fueron becados por alguna institución (una gran cantidad en los últimos años), ayuda que permite a las personas un sitio de trabajo fijo a cambio de cierta cantidad de años, lo cual genera gran seguridad en los becados, pues de otra forma no podrían financiar el curso. Además las instituciones lo ven como una inversión que a futuro les asegura el recurso humano requerido para cubrir las necesidades de la población a su cargo. (Consejo de Salud Ocupacional, 1993)

Es importante recalcar que desde hace varios años son pocas las Áreas de Salud que ofrecen becas para las personas interesadas en realizar el curso y formarse como ATAPS, debido a la disminución en los presupuestos para recurso humano, por ello es que el uso de préstamos con empresas como CONAPE han facilitado a las y los interesados la posibilidad de estudiar. Las cooperativas que siguen ofreciendo becas son COOPESANA, COPESAIN, COOPESALUD y COOPESIBA, entidades que, por el crecimiento en sus áreas, se han visto urgidas a costear este recurso humano en salud.

Acerca del lugar de procedencia, el mayor porcentaje proviene del Área Metropolitana y provincias próximas a ella, como Alajuela, Heredia, Cartago, lo cual se debe a que el curso es impartido en San José, cercanía que representa una ventaja económica y geográfica y que permite a los estudiantes mantenerse cerca de sus hogares y familiares. En contraste, está la población que viene de zonas alejadas del país como Guanacaste, Limón y Puntarenas, pues estas personas deben reubicarse e invertir más dinero para poder formarse como ATAPS; siendo posible solo para aquellos que poseen familiares o amigos cerca del sitio donde se imparte el curso.

Las y los participantes perciben el curso como la única forma de lograr el objetivo propuesto para su superación personal y laboral, objetivo que conlleva mucho esfuerzo, máxime para aquellos que tienen hijos. 
Durante el proceso de la formación, las personas dedican tiempo completo al curso, lo que en muchas ocasiones genera un alto grado de estrés, debido a las exigencias y la necesidad de concluir los estudios técnicos (O' Brien, 1998 y OPS, 2004), debido a que ello repercute en su rendimiento académico, aunque también es un aliciente para cumplir la meta esperada, graduarse y colocarse en alguna área de salud, ya que han invertido tiempo, dinero y algunos refieren sacrificio de sus familiares. (Cases, 2004)

La mayoría de las personas que cursó la formación tuvo un rendimiento acorde con las exigencias del curso, solo dos no se egresaron satisfactoriamente, pues tuvieron calificaciones inferiores al promedio de aprobación (7.0)

Las personas egresadas evalúan el curso de forma satisfactoria, porque obtienen las herramientas para brindar la atención necesaria a los y las usuarias durante la visita domiciliaria con calidad y calidez, no solo tomando los compromisos de gestión como parámetro, sino con el objetivo de disminuir la morbi-mortalidad de la población a su cargo.

El curso de ATAPS que se imparte en la Escuela de Enfermería como proyección social ha permitido que la mayoría de las personas puedan ser parte de la población económicamente activa al estar laborando en las diversas instituciones del país. (Mato, Souza, Cheaz y Santamaria, 2001)

\section{CONCLUSIONES}

- En la actualidad, son pocas las Áreas de Salud de los servicios públicos que han brindado becas a la comunidad para que se formen ATAPS, al respecto se puede afirmar que es el sector privado el que mayormente ofrece ese tipo de ayudas para capacitar a su recurso humano. La Universidad de Costa Rica, por su lado, al ofrecer este curso de ATAPS como parte de un proyecto de Extensión Docente en la Vicerrectoría Acción Social, no ofrece becas a la comunidad, de allí que sean entidades como COONAPE las que brinden el financiamiento.

- En el presente ha aumentado el desempleo, además el mercado laboral se ha saturado, situación que impide a los distintos profesionales ejercer su labor, esto ha llevado a que busquen alternativas para poder insertarse en la población económicamente activa del país.

- En ocasiones cuando la situación económica o personal cambia, las personas consideran que la formación de ATAPS les permite superación personal y estabilidad económica en un corto lapso.

- El rendimiento académico de las personas que se forman como ATAPS es influenciado por situaciones familiares, económicas, reubicación geográfica y situaciones personales.

- El curso ofrece las herramientas generales para poder ejecutar la visita domiciliaria, pilar del perfil del ATAPS, lo cual les permite hacer un diagnóstico de las condiciones de salud de la persona, familia y comunidad.

- La formación brindada por la Escuela de Enfermería de la Universidad de Costa Rica ofrece al país un recurso humano de calidad en materia de atención primaria en salud, el cual posee conocimientos 
técnicos que le permiten brindar una atención integral que mejora la calidad de vida de la población costarricense para así disminuir los índices de morbi-mortalidad regional y nacional.

\section{REFERENCIAS}

Ayala, N; Carvajal, X; Cercome, J y Arce, R. (1999). Curso especial de posgrado. Gestión de la salud: bases del Sistema Nacional de Salud y proceso de modernización de la Caja Costarricense de Seguro Social. San José, Costa Rica.

Arce, P. (1998). Propuesta Residencia Práctica sobre Terapia Familiar Breve. Escuela de Educación Especial. Clínica Infanto Juvenil, Hospital Calderón Guardia. Experiencia con tres familias. Documento para el curso de Residencia Práctica. Maestría en Trabajo Social. Universidad de Costa Rica.

Barrantes, CM. (2000). Programa de educación Permanente dirigido a los Asistentes Técnicos de Atención Primaria, COOPESIBA R.L. Práctica dirigida para optar por el grado de Licenciatura en Enfermería. Universidad de Costa Rica.

Caja Costarricense de Seguro Social. (2009). Dirección de compra de servicios. Recuperado de http://www.ccss.sa.cr/html/organizacion/gestion/gerencias/administrativa/dcss/10a dess.html

Cases, E. (2004). Diferentes forma de respuesta ante el estrés. Recuperado desde: http://www.es.Catholic.net

Consejo de Salud Ocupacional. (1993). Antología. Salud Ocupacional. San José, Costa Rica: CSO

Escuela de Enfermería. (2009). Programa de educación permanente de la Escuela de Enfermería. Programa de Extensión Docente. Vicerretoría de Acción Social. Proyecto número 1539. Universidad de Costa Rica.

Garro, M. (1997). Programa de capacitación dirigido a Asistentes Técnicos de Atención Primaria para el manejo del individuo con trastorno mental en la comunidad, Guápiles. Práctica dirigida para optar por el grado de Licenciatura en Enfermería con énfasis en Salud Mental. Universidad de Costa Rica.

González, E. (1992). Programa de capacitación al personal de atención primaria para reducir la morbimortalidad en la población de 0-6 años, San Carlos 1992. Tesis de la Escuela de Enfermería, Universidad de Costa Rica.

Guillén, AM. (1997). Regímenes de bienestar y roles familiares: un análisis del caso español. Recuperado de http://www.intersindical.org/saludlaboral/stepv/slgenero

Jara, O. (1998). Para Sistematizar Experiencias. San José, Costa Rica: Editorial Alforja.

Jiménez, O., Montero, M., Sánchez, O y Tenorio , V. (2005). Estudio sobre los factores psicosociales intra y extralaborales en los (as) trabajadores (as) de la Compañia Nacional de Fuerza y Luz, edificio Central I semestre 2005. Práctica de investigación aplicada para optar por el grado de Magíster de Enfermería en Salud Laboral. Sistema de Estudios de Posgrado. Universidad de Costa Rica.

Lagarde, M. (1992). Identidad de Género. Managua, Nicaragua: Olof Palme. 
Mato, M.,Souza, J., Cheaz, J. y Santamaria, J. (2001). La Dimensión de Gestión en la construcción de la sostenibilidad institucional. Serie innovación para la innovación institucionalidad. San José, Costa Rica: ISNAR nuevo paradigma.

Méndez, E. y Parada, N.(2001). Informe de investigación sobre necesidades de capacitación en los Asistentes Técnicos de Atención Primaria (ATAPS). Rev. cienc. adm. financ. segur. soc. [online]. jun. 2001, vol.9, no.1 p.53-69. Recuperado de http://www.scielo.sa.cr/scielo.php?script=sci arttext\&pid=S140912592001000100005\&lng=es\&nrm=iso

Montero, M. y Quirós M. (2003). Programa para el desarrollo Personal dirigido a las y los estudiantes del curso de Asistentes Técnicos de Atención Primaria que laborarán en las áreas de salud de ASEMECO. Práctica dirigida para optar por el grado de Licenciatura en Enfermería con énfasis en Salud Mental. Universidad de Costa Rica.

O' Brien, G. (1998). El estrés laboral como factor determinante de la salud. Madrid, España: Buendía Editores.

Organización Panamericana de la Salud. (2004). Perfil del sistema de salud de Costa Rica. Ministerio de Salud de Costa Rica: OPS.

Organización Panamericana de la Salud. (2004). Situación actual de la salud mental en Costa Rica. Ministerio de Salud de Costa Rica: OPS.

Proyecto Estado de la Nación. (2005). Undécimo Informe del Estado de la Nación en Desarrollo Humano Sostenible. San José, Costa Rica.

Vargas, W. (2006). Atención Primaria de Salud en Acción. Su contexto histórico, naturaleza y organización en Costa Rica. Caja Costarricense de Seguro Social y el Centro Estratégico para el Desarrollo e Información en Salud y Seguridad Social. San José, Costa Rica. 